\section{Implant location technique}

Since the publication of our article on removing non-palpable contraceptive implants, ${ }^{1}$ a number of questions have been asked about 'appropriate ultrasound settings and transducers' to use when locating deeply inserted implants. Many contraceptive services in the UK have purchased small, portable ultrasound scanners with high frequency, linear array transducers $(10-15 \mathrm{mHz})$ that are appropriate for scanning musculosketetal sites but clinicians are still failing to find these 'lost implants'.

Portable scanners are becoming more sophisticated, with image-enhancing software installed to filter out artefacts and sharpen anatomical structures. This software can, however, hinder foreign body detection as the acoustic shadowing is lost. It is important, therefore, to switch off the image-enhancing software as illustrated in Figures 1 and 2. By disabling this software, the echogenic 'spot' of the implant can be seen together with the characteristic acoustic shadowing underneath the implant.

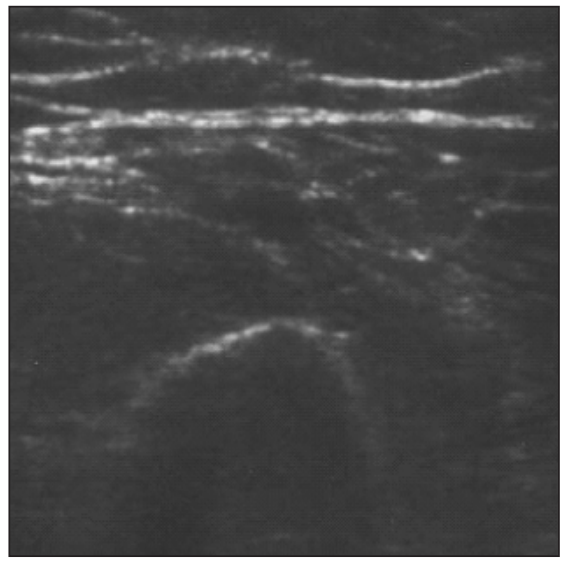

Figure 1 Scan with the image-enhancing software switched on

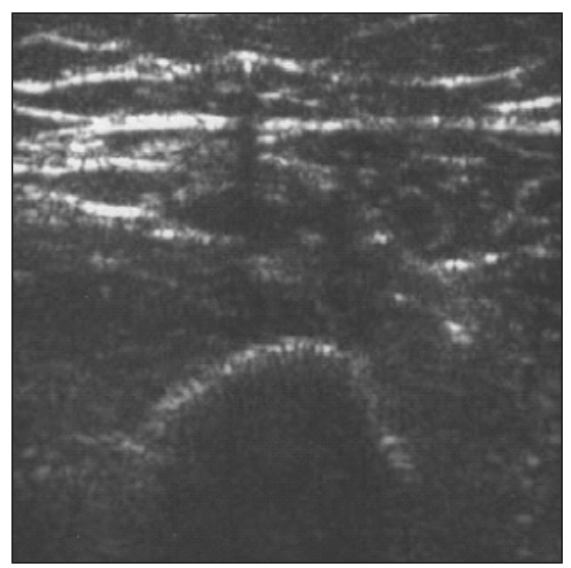

Figure 2 Identical scan with the image-enhancing software switched off

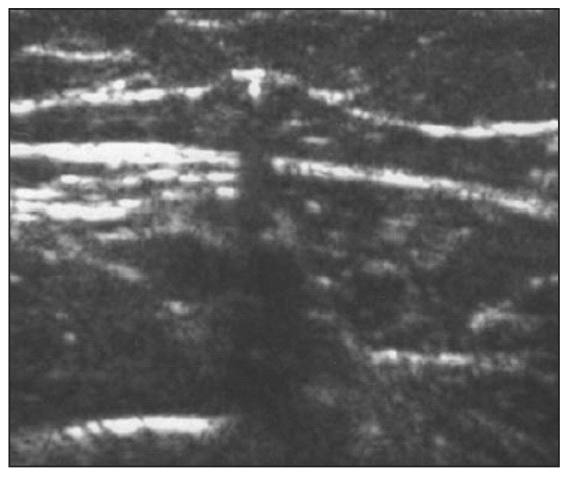
Figure 3 Transverse section of an arm with the image-
enhancing software disabled and the focus set superficially
The depth and the electronic focus should be set as superficially as possible $(1 \mathrm{~cm}$ depth ideally). The image-enhancing software has been switched off in Figure 3 with the depth concentrating on the first centimetre or so below the skin surface, making the transverse section of the implant easy to see and the acoustic shadow obvious

\section{Diana Mansour, FRCOG, FFSRH}

Consultant in Community Gynaecology and

Reproductive Health Care, Sexual Health

Services, New Croft Centre, Newcastle upon

Tyne, UK.

E-mail:diana.mansour@newcastle-pct.nhs.uk

\section{Reference}

Mansour D, Walling M, Glenn D, Egarter C, Graesslin $\mathrm{O}$, Herbst $\mathrm{J}$, et al. Removal of non-palpable etonogestrel implants. J Fam Plann Reprod Health Care 2008; 34: 89-91.

\section{Pregnancy at time of change of Implanon ${ }^{\circledR}$ implant}

The patient had her first Implanon ${ }^{\circledR}$ fitted in her left arm at the age of 23 years when she was 9 weeks postnatal. In the first year she experienced some irregular bleeding and in the second and third years had no bleeding. Her second Implanon was fitted 3 days before the end of the implant's recommended 3-year lifespan and was positioned in a new site in the left arm over the biceps. During the lifespan of this second Implanon the patient experienced regular bleeds every 28-30 days. In 2009, 2 weeks before the 3 year period was up, the patient attended for removal and refit of her implant. The Implanon was removed and a new one fitted through the excision hole

Six weeks after the third Implanon was fitted the patient rang to say she had not had any bleeding since the implant was fitted and that she felt pregnant; in addition, a self-administered pregnancy test was positive. The patient was seen in clinic the next day, when an ultrasound scan revealed an 8-week fetus (crown-rump length, $15.6 \mathrm{~mm}$ ). The patient and her partner decided to continue with this pregnancy and the Implanon was removed.

Over the course of the 6 years that the patient had relied on Implanon for contraception her weight had increased from 84 to $91 \mathrm{~kg}$. She had not taken any drugs, either herbal or prescriptiononly medicines.

Organon Pharmacovigilence have been informed. ${ }^{1}$ Obviously in this particular case it was not possible to take blood samples in order to measure etonogestrel levels.

It is interesting that the patient had regular bleeds with her second Implanon, which differed from the bleeding pattern she experienced with the first Implanon. Might she have been ovulating regularly and been relying on the cervical mucus effect, which was lost for a time at the change of Implanon thus allowing a pregnancy? Why was the bleeding pattern so different with the second Implanon? Should health professionals routinely advise condom use for 7 days following Implanon replacement?

\section{Carole A Reader, MBBCh, DFSRH}

Associate Specialist, Contraception and

Sexual Health, Gloucestershire Royal Hospital,

Gloucester, UK.

E-mail: carole.reader@glos.nhs.uk

\section{Reference}

Mansour D. Implanon failure or natural event? J Fam Plann Reprod Health Care 2007; 33: 127.

\section{Reply}

With regard to the pregnancy at the change of Implanon ${ }^{\circledR}$ reported by $\mathrm{Dr}$ Reader, while a number of pregnancies have been reported with
Implanon, most of these have been linked to noncompliance with the Implanon insertion protocol. ${ }^{2-5}$ Some cases have been reported in which the implant was not inserted on the correct day, or was not properly inserted or not inserted at all. 5 The occurrence of such incidents can be minimised when the instructions for insertion are strictly followed. 5 In the case reported by $\mathrm{Dr}$ Reader, it is not clear whether the pregnancy occurred on the second or third implant. Further, etonogestrel quantification may have helped to assess this case further.

From Dr Reader's comments, it appears that neither herbal nor prescription-only medicines could account for the pregnancy. However, alcohol consumption is not reported; chronic alcohol consumption could have an impact on the efficacy of Implanon as alcohol is a liver enzymeinducer and it may be a confounder or alternative explanation in these cases.

Dr Reader reports that over the course of the 6 years the patient's weight changed from 84 to $91 \mathrm{~kg}$. The contraceptive effect of Implanon is related to the plasma levels of etonogestrel, which are inversely related to body weight, and decrease with time after insertion.5,6 This might explain the change in bleeding pattern for this particular woman with the second implant. In other words, the increase in weight might have exposed her to different etonogestrel levels than those she experienced when using her first implant. Further, clinical experience with Implanon in heavier women in the third year of use is limited. 5 Consequently, it cannot be excluded that the contraceptive effect in these women during the third year of use may be lower than for women of normal weight. Clinicians may therefore consider earlier replacement of the implant in heavier women 5

After the removal of the implant, immediate insertion of another implant will result in continued contraceptive protection. 5 After Implanon insertion, etonogestrel is rapidly absorbed into the circulation. Ovulation-inhibiting concentrations are reached within 1 day. 5,6 During pharmacokinetic studies, when Implanon was inserted on Days $1-5$ of the menstrual cycle, etonogestrel levels sufficient to provide effective contraception were achieved for most women within 8 hours of insertion. ${ }^{6}$ Thus, the Summary of Product Characteristics (SPC) for Implanon does not recommend an additional contraceptive method at change of implant. However, because Implanon does not protect against sexually transmitted infections, condom use is recommended where appropriate.

Boshi Mohlala, MBChB, DFSRH

Medical Adviser Women's Health, Schering

Plough Ltd, Welwyn Garden City, UK.

E-mail:boshi.mohlala@spcorp.com

Florence Falowo, BSc, MSc

Medical Information Officer, Schering-Plough Ltd, Welwyn Garden City, UK

\section{References}

Reader CA. Pregnancy at time of change of Implanon implant (Letter). J Fam Plann Reprod Health Care 2009; 35: 265.

Cooling H, Pauli H. Full-term pregnancy with Implanon ${ }^{\circledast}$ in situ (Letter). J Fam Plann Reprod Health Care 2006; 32: 204.

3 Patni S, Ebden P, Kevelighan E, Bibby J. Ectopic pregnancy with Implanon" prescription-only medicines. $J$ Fam Plann Reprod Health Care 2006; 32: 115.

Harrison-Woolrych M, Hill R. Unintended pregnancies with the etonogestrel implant (Implanon): a case series with the etonogestrel implant (Implanon): a case series from postmarketing experience

Contraception 2005, 71: 306-308.

Organon Laboratories Ltd. Summary of Product Characteristics: Implanon $68 \mathrm{mg}$ implant for subdermal use. http://emc.medicines.org.uk/document.aspx? documentld=5382 [Accessed 27 August 2009].

6 Bennink HJ. The pharmacokinetics and pharmacodynamics of Implanon, a single-rod etonogestrel contraceptive implant. Eur J Contracept Reprod Health Care 2000; 5(Suppl. 2): 12-20. 